\title{
Stress analysis and design of cylinder bearings supporting metal bridges
}

\author{
Andrew Ch. Yiannopoulos
}

\begin{abstract}
The present work presents a method for dimensioning solid cylinders supporting metal bridges at the side of the stationary hinge, which is known as cylinder bearing. The hinge constitutes of two plates with curved surfaces in contact with a cylinder between them. The imposed forces on the hinge cause contact pressures distributed on two antisymmetric circular arcs. If the arcs are too large, the Hertz theory can not be used for the calculation of stresses because it gives no sufficient results. For this particular problem an exact solution is given for normal and shearing stresses on the whole cylinder cross section. The octahedral shearing stress is used as a criterion to express the strength of the cylinder according to the maximum distortion energy theory, because it offers a more reliable tool to estimate the strength of the cylinder material. Moreover, a design equation is derived which may be useful to engineers, if they have to calculate the cylinder dimensions. The results show that the cylinder dimensions should be higher than estimated by other designers.
\end{abstract}

Index Terms - Bridge bearings, Contact of conforming surfaces, Solid cylinders, Stress analysis in cylinders.

\section{INTRODUCTION}

An extended reference about the types of bridge bearings and materials is presented in the book by Eggert and Kauschke [1], among them the cylinder bearings used in metal bridges are included and the most common materials may be: - Structural steel S235-J2G3 and S355-J2G3

- Cast steel GS-52.3 and heat-treatable steel C 35

- Stainless forging steel X4 CrNiMo16-5-1

In the same reference one can find the selection of appropriate screws or weld connections as well as the dimensions of bearing plates. The methods of calculating the pressure in the bearing joints and the constructional anchoring to fix the bearings in position are also described. Steel point rocker bearings are examined and the maximum contact pressure is calculated using the Hertz pressure distribution, as well as the equation for the width of the contact area is also given.

Moreover, the types of cylinder bearings and roller bearings which are used in metal bridges are also presented in the book by Mehlhorn and Curbach [2]. Although these types of bearings can undertake vertical forces from 200 to 20.000 $\mathrm{kN}$, in the new technical works are not used too frequently as in the past. Instead of them other types of bearings are usually applied, e.g. bearings with elastomer or PTFE substrates.

It is widely known that the Hertz theory predicts the stresses at positions close to the contact area. This seems very

A. Ch. Yiannopoulos, Department of Mechanical Engineering, Technological Education Institution of Western Greece, M. Alexandrou 1, Patras 26334, Greece, Phone: +30-2610-369084, Fax: +30-2610-369198. important because it applies in contacts between two bodies extended to infinity and that is why the whole shape of finite bodies is not incorporated in the Hertz solution [3]. For the case of contact between the cylinder of a bridge bearing and the conforming surface of the girder or base plate, if the arc of contact between the bodies is not small enough, the analysis of radial displacements differs significantly from Hertz approximation, although the latter is satisfactory for non-conforming contact surfaces, such as a cylinder on a flat plate. Furthermore, it is well established by Johnson [4] that when the arc of contact occupies an appreciable fraction of the circumference of the cylinder neither the cylinder nor the plate surface can be regarded as an elastic half-space so that the Hertz treatment is invalid.

Persson [5] was the first who presented a detailed study for the stress field of a pin and a circular hole in the PhD dissertation. For cylindrical joints with clearance, the Hertz model and the Persson model are the common methods for describing the contact behaviour of the clearance. The Hertz model is preferable in the case that there is a large clearance with a small normal load. The Persson model can be applied in the case that there is a small clearance. Chen and Marshek [6] presented a numerical procedure for solving two-dimensional closely conforming elastic body contact problems and examined the influence of the clearance and material properties on the contact pressure of two cylinders. The numerical results have been verified by comparison with the analytical solution by Persson's. They also showed that the use of Hertz theory gives an underestimation of the peak pressure and overestimation of the subtended angle. The method can be applied in the design of certain mechanical joint elements and aligned journal bearings.

Mostofi and Gohar [7] studied the elastic contact of a long roller pressed against a cylindrical hole of an infinite body by concentrated line forces and determined the pressure distribution, as well as the extent of the contact arc and the deflection of the bodies. They showed that the values of characteristic variables depend on the degree of conformity of the two bodies and that the Hertz theory should not be used when the dimensions of the contact arc are of the same order as the radii of curvature. In such a case a considerable stress concentration occurs at the edges of the arc.

A numerical method for the determination of the contact pressure that arises if two elastic bodies with closely conforming surfaces are pressed together was developed by Paul and Hashemi [8]. They also considered the contact of the wheel and rail of a train in closely conforming region of the flange throat, i.e. at the wheel disk curvature. The work of Wanlin [9] provided numerical solutions for stress intensity factors for corner cracks at holes subjected to biaxial and pin loads. The distribution of the load on the boundary of the hole is always influenced by many factors, such as joint types, materials of the components, fit condition between hole and 
pin, geometry of the plate containing the hole, loading configuration, shape and size of the crack etc. The researcher assumed three kinds of typical distribution for the pin load frequently used in engineering applications, which are concentrated, uniform and with cosine distributions.

Fujii et al. [10] analysed the stress and displacement field around a circular interface crack between a rigid cylinder and an infinite elastic medium and gave the solution by Fourier series. Assuming a concentrated force applying at the center of the cylinder they presented the stress components and displacements along the interface of two bodies in contact. Chiavarella et al. [11] studied the contact problems of almost uniformly bodies and showed that as the applied load is increased the contact area tends asymptotically to the solution of the corresponding receding contact problem, i.e. the case in which the clearance or interference is identically zero. Liu et al. [12] used a finite elements method to approximate model for the above referred cylindrical joints with clearances. They presented numerical results such as the contact area, the pressure distribution and the maximum sustainable load, and they examined the influence of the size of the joint clearance on the relation between the contact load and penetration displacement. They also presented a comparison with Persson's model to verify the precision of results.

The present work is focused on external pressures coming from non-Hertzian contacts acting on the cylinder bridge bearings and predicts the stress components developed inside the whole body of the elastic solid cylinder, if the law of distributed forces and the arcs of application are given. Under this mode of loading the general solution for stress distribution at any point of the cross-section of the cylinder is derived and presented in a simplified form. Then the octahedral shearing stress is calculated and the procedure leads to the estimation of cylinder dimensions, which are sufficient to suffer the applied forces. It is known that the octahedral shearing stress theory is equivalent to the energy of distortion theory, so this stress is associated with material yielding and is wholly responsible for starting inelastic action in the body. Therefore, a criterion of failure can be used which is incorporated with the tensile elastic strength of the material.

Moreover, the method proposed herein, for the analysis of the particular case of a cylinder bridge bearing, is based on the maximum value of octahedral shearing stress which appears inside the body of the cylinder and that is the difference between the present method and Hertz theory. The latter deals with the contact pressures on the outer surface and gives no valid results for the determination of cylinder dimensions, if the clearance between cylinder and hole is too small or the two radii of curvatures are practically equal. Consequently, it may be considered that this point of research is the most significant contribution to the science of engineering.

\section{METHOD OF ANALYSIS}

A stationary hinge of a metal bridge consists of a cylinder between two plates with curved contact surfaces, as shown in Fig. 1. This kind of bridge support is characterised as a cylinder bearing. The cylinder is solid and long with circular cross section. The plates 1 and 2 have circular curvatures with $\operatorname{arcs} 2 \alpha_{1}$ and $2 \alpha_{2}$, and radius of curvature equal to the cylinder radius. It must be noted that it is more convenient to use in the analysis that follows the semi-arcs $\alpha_{1}$ and $\alpha_{2}$ instead of the whole arcs $2 \alpha_{1}$ and $2 \alpha_{2}$, due to symmetry of the pressures. Both arcs are equal $\left(\alpha_{1}=\alpha_{2}\right)$, whereas in practice the whole arcs are usually greater than $90^{\circ}$ and lower enough than $180^{\circ}$.

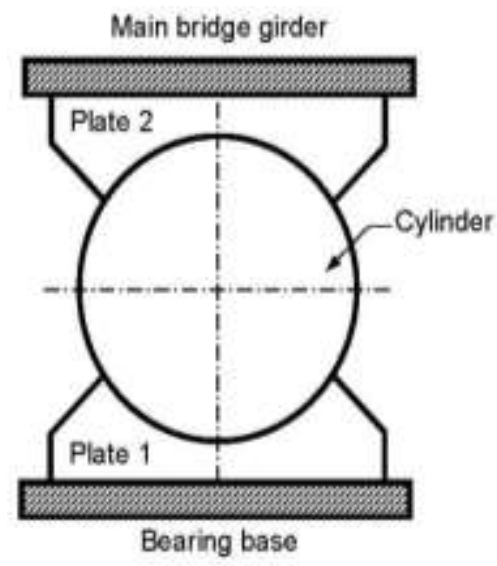

Fig. 1: Metal bridge stationary hinge with a cylinder bearing

The total force $P$ of the main bridge girder acts on plate 2 in the direction of vertical axis and is transferred to the cylinder. It is undertaken by the bearing base of the bridge producing an equal reaction $P$ through plate 1 . These forces act radially on the circular surface of the cylinder along the two arcs of contact. The magnitude of the arcs and the mode of distributed forces depend on the quality of surfaces in contact and mainly how they conform to each other that is from the magnitude of the clearance that exists.

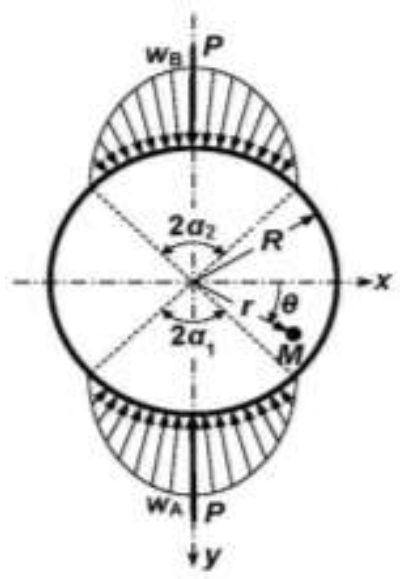

Fig. 2: Cylinder configuration and forces

We consider a non-uniform pressure distribution which is zero at the ends of the arcs and has a maximum value at the center of the arc, as shown in Fig. 2. In this case the most proper mode of distribution for practical applications is the sinusoidal one [9] and the total arc of distributed pressures that may be taking into account for the state of stress should not exceed $90^{\circ}$ even if the actual arc is practically larger [13]. This means that there is an effective arc over which the pressure is transferred to the cylinder. However, in the following the problem is solved in a more general form using some arbitrary values of the $\operatorname{arcs} \alpha_{1}$ and $\alpha_{2}$, and a known maximum force $P$. The distributed forces on the arcs are taken radial having a resultant force in the direction of $y$-axis equal to $P$. Obviously the system is in equilibrium and thus one can write the equation of equilibrium due to symmetry as: 
$P=\frac{4 w_{A} K L}{\pi} \frac{\alpha_{1} \cos \alpha_{1}}{1-4\left(\frac{\alpha_{1}}{\pi}\right)^{2}}=\frac{4 w_{B} K L}{\pi} \frac{\alpha_{2} \cos \alpha_{2}}{1-4\left(\frac{\alpha_{2}}{\pi}\right)^{2}}$

where $\alpha_{1}$ and $\alpha_{2}$ are the semi-arcs of contact, $w_{\mathrm{A}}$ and $w_{\mathrm{B}}$ the pressures at the center of the contact arcs, respectively, $R$ the radius of the cylinder and $L$ the length of the cylinder.

In many common circumstances, the load $P$ is calculated according to the structural design of the bridge and is usually known. In such a case the pressures $w_{\mathrm{A}}$ and $w_{\mathrm{B}}$ on the cylinder with given dimensions $(R, L)$ can be found using (1), if the semi-arcs of contact $\alpha_{1}$ and $\alpha_{2}$ are previously defined.

Starting the analysis of stresses $\sigma_{\mathrm{r}}, \sigma_{\theta}$ and $\tau_{\mathrm{r} \theta}$ it is applied the method of two antisymmetric point forces acting at the positions $\theta= \pm \pi / 2$. For this particular problem two antisymmetric but infinitesimal point forces $\mathrm{d} P$ are assumed and after using a stress function one can reach to the solution of stresses $\mathrm{d} \sigma_{\mathrm{r}}, \mathrm{d} \sigma_{\theta}$ and $\mathrm{d} \tau_{\mathrm{r} \theta}$. This analysis is not given here but is presented in detail in the author's previous work [14]. In the sequel the derived expressions of stresses can be integrated along the arcs of contact supposing a sinusoidal pressure distribution on these arcs and after a lot mathematical manipulations the final expressions of stresses $\sigma_{\mathrm{r}}, \sigma_{\theta}$ and $\tau_{\mathrm{r} \theta}$ are obtained. Some integrals are appeared in the stress equations which are not expressed in analytical form and thus they are calculated through numerical integration. Closing the above procedure, the equations of stresses can be derived in a non-dimensional form, if divided by the term $2 P / \pi R L$. Since the obtained equations are too long, some parts of them are substituted by mathematical functions and then they can be written in a little more compact form. After that the non-dimensional stresses appear as follows.

$$
\begin{aligned}
\frac{\sigma_{\mathrm{r}}}{2 P / \pi R L} & =-\frac{\pi}{4} \frac{1-4\left(\frac{\alpha_{1}}{\pi}\right)^{2}}{\cos \alpha_{1}} \int_{-\frac{\pi}{2}}^{\frac{\pi}{2}} f_{1}(x) \cos \left(\frac{\pi}{2} \sin x\right) \cos x \mathrm{~d} x-\frac{\pi}{4} \frac{1-4\left(\frac{\alpha_{2}}{\pi}\right)^{2}}{\cos \alpha_{2}} \int_{-\frac{\pi}{2}}^{\frac{\pi}{2}} f_{2}(x) \cos \left(\frac{\pi}{2} \sin x\right) \cos x \mathrm{~d} x \\
\frac{\sigma_{\theta}}{2 P / \pi R L} & =-\frac{\pi}{4} \frac{1-4\left(\frac{\alpha_{1}}{\pi}\right)^{2}}{\cos \alpha_{1}} \int_{-\frac{\pi}{2}}^{\frac{\pi}{2}} f_{1}(x) \cos \left(\frac{\pi}{2} \sin x\right) \cos x d x-\frac{\pi}{4} \frac{1-4\left(\frac{\alpha_{2}}{\pi}\right)^{2}}{\cos \alpha_{2}} \int_{-\frac{\pi}{2}}^{\frac{\pi}{2}} f_{4}(x) \cos \left(\frac{\pi}{2} \sin x\right) \cos x \mathrm{~d} x \\
\frac{\tau_{\mathrm{r} \theta}}{2 P / \pi R L} & =\frac{\pi}{4} \frac{1-4\left(\frac{\alpha_{1}}{\pi}\right)^{2}}{\cos \alpha_{1}} \int_{-\frac{\pi}{2}}^{\frac{\pi}{2}} f_{5}(x) \cos \left(\frac{\pi}{2} \sin x\right) \cos x \mathrm{~d} x-\frac{\pi}{4} \frac{1-4\left(\frac{\alpha_{2}}{\pi}\right)^{2}}{\cos \alpha_{2}} \int_{-\frac{\pi}{2}}^{\frac{\pi}{2}} f_{6}(x) \cos \left(\frac{\pi}{2} \sin x\right) \cos x \mathrm{~d} x
\end{aligned}
$$

where $\xi=r / R$ is the dimensionless radius, $(r, \theta)$ the polar coordinates of the cross section, $P$ the total force, $R$ and $L$ the radius and length of the cylinder, respectively. The angle $\theta$ is measured from $x$-axis to the clock-wise direction (see Fig. 2). The functions $f_{1}(x), f_{2}(x), f_{3}(x), f_{4}(x), f_{5}(x)$, and $f_{6}(x)$ are given in the Appendix.

Since the length of the cylinder is large comparing with the diameter it can be assumed that a two-dimensional stress problem of plane-strain exists. For such a problem, the two principal stresses $\sigma_{1}$ and $\sigma_{2}$ can be expressed through the components $\sigma_{\mathrm{r}}$ and $\sigma_{\theta}$, respectively. Furthermore, the third principal stress $\sigma_{3}$, which is produced in the direction of $z$-axis that is along the length of the cylinder, can be calculated according to the Poisson's theory and introducing the ratio $v$, as:

$$
\sigma_{\mathrm{z}}=v\left(\sigma_{\mathrm{r}}+\sigma_{\theta}\right)
$$

Getting the solution of stresses $\sigma_{\mathrm{r}}, \sigma_{\theta}, \sigma_{\mathrm{z}}$ and $\tau_{\mathrm{r} \theta}$, it is easy to find the magnitude of the octahedral shearing stress $\tau_{\mathrm{G}}$, which is expressed in polar coordinates through the following equation [3]:

$\tau_{\mathrm{G}}=\frac{\sqrt{2}}{\mathrm{a}} \sqrt{\sigma_{\mathrm{r}}^{2}+\sigma_{\theta}^{2}+\sigma_{\mathrm{z}}^{2}-\sigma_{\mathrm{r}} \sigma_{\theta}-\sigma_{\theta} \sigma_{\mathrm{z}}-\sigma_{\mathrm{z}} \sigma_{\mathrm{r}}+3 \tau_{\mathrm{r} \theta}^{2}}$
The latter equation can be transformed in order to produce a dimensionless part, denoted by $\chi_{\mathrm{G}}$, if it is divided by the term $2 P / \pi R L$, and then it can be written in a simplified form as follows:

$$
\frac{\tau_{\mathrm{G}}}{2 P / \pi R L}=\chi_{\mathrm{G}}
$$

It is very important to note that $\chi_{\mathrm{G}}$ is a dimensionless parameter depending only on the geometry of the stress problem. Continuing the procedure an appropriate criterion should be used for the strength of the cylinder material, through which the cylinder dimensions can be determined according to the applied force $P$ on the bridge bearing. This criterion is expressed through the following equation [3]:

$$
\tau_{\mathrm{G}}=\frac{\sqrt{2}}{3} \frac{R_{\mathrm{e}}}{N}
$$

where $R_{\mathrm{e}}$ is the maximum elastic strength of the cylinder material due to tension (yield strength) and $N$ the safety factor that exists. Combining (5) and (6) and introducing the cylinder diameter $d$ instead of radius $R$, the final solution of the problem is obtained after solving for $d$, as: 


$$
d=\frac{6 \sqrt{2}}{\pi} \chi_{\mathrm{G}} \frac{P}{L R_{\mathrm{e}} / N}
$$

In practical cases it is more convenient to use $q$ as the load per unit length, so the ratio $P / L$ can be substituted in (7) by $q$. Furthermore, inserting the suggested factor of safety $N=2.5$ for cylinder bearings in the bridge hinges [15], a more simplified form of the final design equation is taken, which may be written in SI units as:

$$
d=6.75 \chi_{\mathrm{G}} \frac{q}{R_{\Theta}}
$$

where $d$ is the cylinder diameter, in $\mathrm{mm} ; \chi_{\mathrm{G}}$ the maximum value of dimensionless parameter of octahedral shearing stress; $q$ the load per unit length acting on the cylinder bearing, in $\mathrm{kN} \cdot \mathrm{m}^{-1} ; R_{\mathrm{e}}$ the yield strength of the material, in $\mathrm{N} \cdot \mathrm{mm}^{-2}$.

It is worth noting that the proposed method has some substantial advantages, because it uses no Fourier series in the expressions of stresses and thus eliminates any problem of convergence. Therefore, there is no limitation and exact stress values may be computed near the boundary, where the method through a Fourier expansion of the applied pressures would have serious difficulties. Moreover, the most important is that the maximum value of the parameter $\chi_{\mathrm{G}}$, for a given Poisson's ratio $v$, depends only on the geometry of the problem and actually on the contact arcs $\left(\alpha_{1}\right.$ and $\left.\alpha_{2}\right)$, so it may be ready for use if tabulated or plotted in graphs.

\section{NUMERICAL EXAMPLE AND DISCUSSION}

As a numerical example, it is solved the problem of a cylinder with an effective length $L=400 \mathrm{~mm}$ and a total force $P=2000 \mathrm{kN}$, which is equivalent with a load per unit length $q$ $=5000 \mathrm{kN} \cdot \mathrm{m}^{-1}$. The Poisson's ratio is taken $v=0.3$. The calculation of octahedral shearing stress is performed for many values of semi-arcs $\alpha_{1}$ and $\alpha_{2}$, namely $20^{\circ}, 30^{\circ}, 40^{\circ}, 45^{\circ}$ and $60^{\circ}$. As it is suggested before, the effective semi-arc of contact should not exceed the value $45^{\circ}$, even if the contact is larger for the reason explained in the previous section. Thus, the value of $45^{\circ}$ is applied in (1) and after doing the necessary manipulations the total force $P$ can be written in the form:

$$
P=0.943 w_{\mathrm{A}} R L
$$

The latter equation makes it possible to calculate $w_{\mathrm{A}}$ from $P$ if it is desirable. Since $w_{\mathrm{A}}$ is the maximum acting pressure, one can define the dimensions $(R, L)$ of the cylinder if $w_{\mathrm{A}}$ must be lower than the allowable pressure. This approach is followed by some designers but it is not crucial. The author's procedure is completely different and is outlined in brief into the following three steps:

Step 1: The non-dimensional values of stresses $\sigma_{\mathrm{r}}, \sigma_{\theta}, \sigma_{\mathrm{z}}, \tau_{\mathrm{r} \theta}$ of the cylinder cross section are obtained through (2). This is accomplished by solving the right part of their expressions for many points $(\xi, \theta)$ of the cross section using the given values of semi-arcs $\alpha_{1}$ and $\alpha_{2}$.
Step 2: The results of stresses are introduced in (4), and then the dimensionless value of $\chi_{\mathrm{G}}$ is extracted for each point $(\xi, \theta)$, as it is denoted by (5). After that the maximum value of $\chi_{\mathrm{G}}$ is searched.

Step 3: The cylinder diameter is calculated using the design equation (8).

The analysis shows that the maximum $\chi_{\mathrm{G}}$ occurs at the $y$-axis, i.e. the vertical axis of symmetry of the cylinder cross section. For small contact arcs the maximum value appears near the surface, whereas if the arcs become larger it moves to the center of the cylinder. This result is expected because the problem with small arcs tends to be somewhat similar to a Hertz contact problem, for which the shearing stress is greater in a small depth under the surface of contact than it is on the surface itself or in greater depths [16].

The behaviour of $\chi_{\mathrm{G}}$ is clearly presented in Fig. 3, where the curves show the variation for five characteristic values of semi-arcs. The critical values of $\chi_{\mathrm{G}}$ and the corresponding points of the curves are listed in Table I. A limited number of values $\chi_{\mathrm{G}}$ for semi-arcs $45^{\circ}$ is also included in Table II.

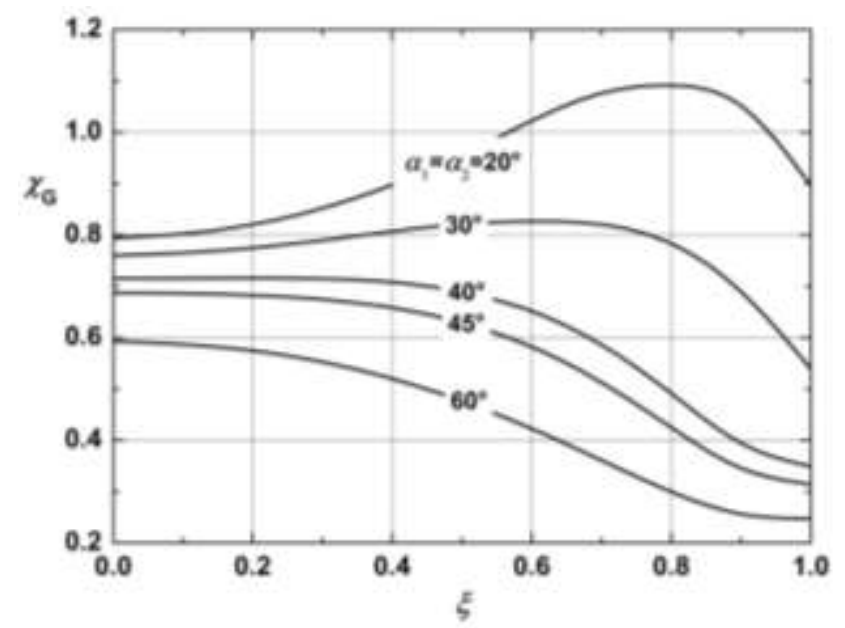

Fig. 3: Dimensionless parameter $\chi_{\mathrm{G}}$ on the $y$-axis as a function of cylinder radius $\xi$ and arc of contact (for $v=0.3$ )

Table I. Critical values of $\chi_{G}$

\begin{tabular}{ccc}
\hline $\begin{array}{c}\text { Semi-arc of } \\
\text { contact } \\
\alpha_{1}=\alpha_{2}\end{array}$ & $\begin{array}{c}\text { Maximum } \\
\text { value } \\
\chi_{\mathrm{G}}\end{array}$ & $\begin{array}{c}\text { Position } \\
\xi\end{array}$ \\
\hline $20^{\circ}$ & 1.096 & 0.75 \\
$30^{\circ}$ & 0.827 & 0.56 \\
$40^{\circ}$ & 0.716 & 0.20 \\
$45^{\circ}$ & 0.687 & 0 \\
$60^{\circ}$ & 0.593 & 0 \\
\hline
\end{tabular}

To illustrate the proposed method and make it more comprehensible, the arithmetic calculation for two different materials, e.g. a cast steel GS-52 and a heat treated steel C35, is presented below. Their limit values of yielding $R_{\mathrm{e}}$, which are valid for $40 \mathrm{~mm}<d \leq 100 \mathrm{~mm}$, are as follows [17]:

$$
\text { GS-52 } R_{\mathrm{e}}=260 \mathrm{~N} \cdot \mathrm{mm}^{-2} \text { (according to DIN 1681) }
$$$$
\text { C35 } R_{\mathrm{e}}=320 \mathrm{~N} \cdot \mathrm{mm}^{-2} \text { (according to DIN EN } 10083 \text { ) }
$$ 
The semi-arcs of contact $\alpha_{1}$ and $\alpha_{2}$ are taken $45^{\circ}$ and therefore the maximum value of $\chi_{\mathrm{G}}$ is 0.687 (see Table I). Applying the data to the design equation (8), the following values of cylinder diameter are obtained:

GS-52: $d=6.75 \times 0.687 \times \frac{5000 \mathrm{kN} \cdot \mathrm{m}^{-1}}{260 \mathrm{~N} \cdot \mathrm{mm}^{-2}}=89.2 \mathrm{~mm} \approx 90 \mathrm{~mm}$

C35:

$d=6.75 \times 0.687 \times \frac{5000 \mathrm{kN} \cdot \mathrm{m}^{-1}}{\mathrm{a} 20 \mathrm{~N} \cdot \mathrm{mm}^{-2}}=72.5 \mathrm{~mm} \approx 75 \mathrm{~mm}$

Table II. Values of $\chi_{\mathrm{G}}$ with respect to $\xi$ and $\theta$ (for $\alpha_{1}=\alpha_{2}=45^{\circ}, v=0.3$ )

\begin{tabular}{cccccccccc}
\hline \multirow{2}{*}{ Position $\xi \xi$} & \multicolumn{1}{c}{ Angle $\theta$} \\
\cline { 2 - 10 } & $-90^{\circ}$ & $-70^{\circ}$ & $-50^{\circ}$ & $-30^{\circ}$ & 0 & $30^{\circ}$ & $50^{\circ}$ & $70^{\circ}$ & $90^{\circ}$ \\
\hline 0 & 0.687 & 0.687 & 0.687 & 0.687 & 0.687 & 0.687 & 0.687 & 0.687 & 0.687 \\
0.2 & 0.683 & 0.678 & 0.665 & 0.649 & 0.638 & 0.649 & 0.665 & 0.678 & 0.683 \\
0.4 & 0.660 & 0.644 & 0.599 & 0.543 & 0.500 & 0.543 & 0.599 & 0.644 & 0.660 \\
0.6 & 0.586 & 0.565 & 0.493 & 0.385 & 0.313 & 0.385 & 0.493 & 0.565 & 0.586 \\
0.8 & 0.425 & 0.403 & 0.337 & 0.193 & 0.132 & 0.193 & 0.337 & 0.403 & 0.425 \\
0.95 & 0.308 & 0.249 & 0.138 & 0.044 & 0.027 & 0.044 & 0.138 & 0.249 & 0.308 \\
0.99 & 0.310 & 0.238 & 0.063 & 0.008 & 0.005 & 0.008 & 0.063 & 0.238 & 0.310 \\
1.0 & 0.314 & 0.241 & 0.055 & 0 & 0 & 0 & 0.055 & 0.241 & 0.314 \\
\hline
\end{tabular}

It is found in the literature that for the case of cylinder bearings of metal bridges, some designers suggest a much more simplified method, but not accurate enough, in order to determine the dimensions of the cylinder [13], [18]. They assume that the total force $P$ has a sinusoidal pattern of distribution over both antisymmetric arcs of $90^{\circ}$ and then they proceed with an equation which is the same as (9). It is clear that this method deals only with the force $P$ and the peak contact pressure $w_{\mathrm{A}}$ on the outer surface, and not with the stress field itself in the whole cross section. The criterion they used for the material strength is expressed by the law which states that the peak pressure $w_{\mathrm{A}}$ should be lower than the allowable contact pressure $\sigma_{\mathrm{zul}}$. The latter is considered equal to the allowable stress for bending and the following values of $\sigma_{\text {zul }}$ are suggested:

$$
\begin{array}{ll}
\text { GS-52: } & \sigma_{\text {zul }}=180 \mathrm{~N} \cdot \mathrm{mm}^{-2} \\
\text { C35: } & \sigma_{\text {zul }}=200 \mathrm{~N} \cdot \mathrm{mm}^{-2}
\end{array}
$$

Applying these values of $\sigma_{\text {zul }}$ for $w_{\mathrm{A}}$ in (9) and the same data of $P$ and $L$ as previous, the corresponding values of cylinder diameter are obtained:

GS-52:

$$
d=\frac{2 \times 2000 \times 10^{8} \mathrm{~N}}{0.943 \times 400 \mathrm{~mm} \times 180 \mathrm{~N} \cdot \mathrm{mm}^{-2}}=58.9 \mathrm{~mm} \approx 60 \mathrm{~mm}
$$

C35:

$$
d=\frac{2 \times 2000 \times 10^{8} \mathrm{~N}}{0.943 \times 400 \mathrm{~mm} \times 200 \mathrm{~N} \cdot \mathrm{mm}^{-2}}=53.0 \mathrm{~mm} \approx 55 \mathrm{~mm}
$$

By comparing the results it is noticed that the method proposed herein predicts much larger dimensions for the cylinder of the bridge bearing, consequently the whole structure will be safer.

\section{CONCLUSION}

The distribution of stresses in a solid cylinder of a bridge bearing is successfully analyzed and presented in a simplified form for any arc of distributed forces on the cylindrical surface. The values of the octahedral shearing stress are obtained analytically, the maximum value of which is used to examine the strength of the cylinder material through a criterion according to the distortion energy theory. In this way the difficulties or inaccuracies have been overcome in contrast to other methods, which use the pressures only on the outer surface and not take into account the stress distribution over the cross section.

However, as the most remarkable conclusion and the main innovation of the present work, it may be considered the derivation of a design equation for the diameter of the cylinder. This equation involves a dimensionless parameter of the octahedral shearing stress, the maximum value of which depends only on the arc of contact and may be given in tables or graphs, in order to be convenient to engineers for using it in the design equation. Moreover, the results of this work may be useful to designers, if they have to estimate the permissible loads for cylinders used in a variety of engineering structures and particularly in bearings of metal bridges, where contact pressures exist with known loading arcs and forces. The comparison of results with those of a different method used from other designers shows that the proposed method is more accurate giving much larger dimensions and thus the aforementioned structures will operate safer having long life.

\section{APPENDIX}

The functions used in the analysis have the following forms: 


$$
\begin{aligned}
& f_{1}(x)=\frac{\left[1-\xi \sin \left(\theta-\alpha_{1} \sin x\right)\right]\left[\xi-\sin \left(\theta-\alpha_{1} \sin x\right)\right]^{2}}{\left[1+\xi^{2}-2 \xi \sin \left(\theta-\alpha_{1} \sin x\right)\right]^{2}}-\frac{1-\sin \left(\theta-\alpha_{1} \sin x\right)}{4} \\
& f_{2}(x)=\frac{\left[1+\xi \sin \left(\theta-\alpha_{2} \sin x\right)\right]\left[\xi+\sin \left(\theta-\alpha_{2} \sin x\right)\right]^{2}}{\left[1+\xi^{2}+2 \xi \sin \left(\theta-\alpha_{2} \sin x\right)\right]^{2}}-\frac{1+\sin \left(\theta-\alpha_{2} \sin x\right)}{4} \\
& f_{1}(x)=\frac{\cos ^{2}\left(\theta-\alpha_{1} \sin x\right)\left[1-\xi \sin \left(\theta-\alpha_{1} \sin x\right)\right]}{\left[1+\xi^{2}-2 \xi \sin \left(\theta-\alpha_{1} \sin x\right)\right]^{2}}-\frac{1+\sin \left(\theta-\alpha_{1} \sin x\right)}{4} \\
& f_{4}(x)=\frac{\cos ^{2}\left(\theta-\alpha_{2} \sin x\right)\left[1+\xi \sin \left(\theta-\alpha_{2} \sin x\right)\right]}{\left[1+\xi^{2}+2 \xi \sin \left(\theta-\alpha_{2} \sin x\right)\right]^{2}}-\frac{1-\sin \left(\theta-\alpha_{2} \sin x\right)}{4} \\
& f_{5}(x)=\frac{\left.\cos \left(\theta-\alpha_{1} \sin x\right)\left\lfloor 1-\xi \sin \left(\theta-\alpha_{1} \sin x\right)\right\rfloor \xi-\sin \left(\theta-\alpha_{1} \sin x\right)\right\rfloor}{\left[1+\xi^{2}-2 \xi \sin \left(\theta-\alpha_{1} \sin x\right)\right]^{2}}-\frac{\cos \left(\theta-\alpha_{1} \sin x\right)}{4} \\
& f_{6}(x)=\frac{\left.\cos \left(\theta-\alpha_{2} \sin x\right)\left\lfloor 1+\xi \sin \left(\theta-\alpha_{2} \sin x\right)\right\rfloor \xi \xi \sin \left(\theta-\alpha_{2} \sin x\right)\right\rfloor}{\left[1+\xi^{2}+2 \xi \sin \left(\theta-\alpha_{2} \sin x\right)\right]^{2}}-\frac{\cos \left(\theta-\alpha_{2} \sin x\right)}{4}
\end{aligned}
$$

\section{NOMENCLATURE}

The following symbols are used in this paper:

$d=$ cylinder diameter

$L=$ cylinder length

$N=$ safety factor

$P=$ resultant force on the cylinder

$q=$ load per unit length

$R=$ cylinder radius

$R_{\mathrm{e}}=$ yield strength

$(r, \theta)=$ polar coordinates

$w_{\mathrm{A}}, w_{\mathrm{B}}=$ peak pressures of contact

$x, y, z=$ orthogonal coordinates

Greek Symbols

$\alpha_{1}, \alpha_{2}=$ semi-arc of contact

$v=$ Poisson's ratio

$\xi=$ dimensionless radius $(=r / R)$

$\sigma_{1}, \sigma_{2}, \sigma_{3}=$ principal stresses

$\sigma_{\mathrm{r}}=$ radial stress

$\sigma_{\theta}=$ hoop stress

$\sigma_{\mathrm{z}}=$ normal stress in $z$-axis

$\sigma_{\mathrm{zul}}=$ allowable compressive stress

$\tau_{\mathrm{G}}=$ octahedral shearing stress

$\tau_{\mathrm{r} \theta}=$ shearing stress

$\chi_{\mathrm{G}}=$ dimensionless octahedral shearing stress

Abbreviations

PTFE $=$ Polytetrafluoroethylene (Teflon)

\section{REFERENCES}

[1] H. Eggert, and W. Kauschke, Structural Bearings. Ernst \& Sohn, Berlin, 2002

[2] G. Mehlhorn, and M. Curbach, Handbuch Brücken. 3. Auflage, Springer Vieweg, 2014 (in German).

[3] F. B. Seely, and J. O. Smith, Advanced Mechanics of Materials. $2^{\text {nd }}$ Edition, John Wiley \& Sons, Inc., U.S.A., 1952.
[4] K. L. Johnson, Contact Mechanics. Cambridge University Press, 1985

[5] A. Persson, "On the stress distribution of cylindrical elastic bodies in contact". PhD Dissertation, Chalmers University of Technology, Gothenburg, Sweden, 1964.

[6] H. H. Chen, and K. M. Marshek, "Effect of clearance and material property on contact pressure in two-dimensional conforming cylinders". Mech Mach Theory, Vol. 23, No. 1, pp. 55-62, 1988.

[7] A. Mostofi, and R. Gohar, "Pressure distribution between closely contacting surfaces". J Mech Eng Sci., Vol. 22, No. 5, pp. 251-259, 1980.

[8] B. Paul, and J. Hashemi, "Contact pressures on closely conforming elastic bodies". J Appl Mech., Vol. 48, pp. 543-548, 1981.

[9] G. Wanlin, "Stress intensity factors for corner cracks at holes subjected to biaxial and pin loads". Eng Fract Mech., Vol. 46, No. 3, pp. 473-479, 1993.

[10] K. Fujii, Y. Kato, S. Duan, and K. Nakagawa, "Stress analysis around a partially bonded rigid cylinder in an elastic medium with process zones". Eng Fract Mech., Vol. 45, No. 1, pp. 31-38, 1993.

[11] M. Chiavarella, A. Baldini, J. R. Barber, and A. Strozzi, "Reduced dependence on loading parameters in almost conforming contacts". Int J Mech Sci., Vol. 48, No. 9, pp. 917-925, 2006.

[12] C.-S. Liu, K. Zhang, and R. Yang, "The FEM analysis and approximate model for cylindrical joints with clearances". Mech Mach Theory, Vol. 42, No. 2, pp. 183-197, 2007.

[13] Stahl im Hochbau. Verein Deutscher Eisenhüttenleute, 13. Auflage, Verlag Stahleisen m.b.H., Düsseldorf, 1969 (in German).

[14] A. Ch. Yiannopoulos, "A general formulation of stress distribution in cylinders subjected to non-uniform external pressure". J Elast., Vol. 56, pp. 181-198, 1999.

[15] R. L. Brockenbrough, and F. S. Merritt, Structural Steel Designer's Handbook. $3^{\text {rd }}$ Edition, McGraw-Hill, Inc., USA, 1999.

[16] S. P. Timoshenko, and J. N. Goodier, Theory of Elasticity. $3^{\text {rd }}$ Edition, McGraw- Hill, Inc., New York, 1982.

[17] B. Künne, Maschinenteile 1. 10. Auflage, B.G. Teubner Verlag, Wiesbaden, 2007 (in German).

[18] A. D. Kosteas, Steel Bridges. $5^{\text {th }}$ Edition, Technical Chamber of Greece, Athens, 1972 (in Greek).

A. Ch. Yiannopoulos, PhD, Associate Professor, Technological Education Institution of Western Greece, Dept. of Mechanical Engineering, Patras, Greece. Research work in Steel structures, Elasticity, Thermoelasticity and Heat transfer. 